\title{
Is the $\sigma_{2}$ Receptor a Histone Binding Protein?
}

Nicola Antonio Colabufo,* Francesco Berardi, Carmen Abate, Marialessandra Contino, Mauro Niso, Roberto Perrone.

Dipartimento Farmacochimico, Università degli Studi di Bari, via Orabona 4, 70125, Bari, Italy

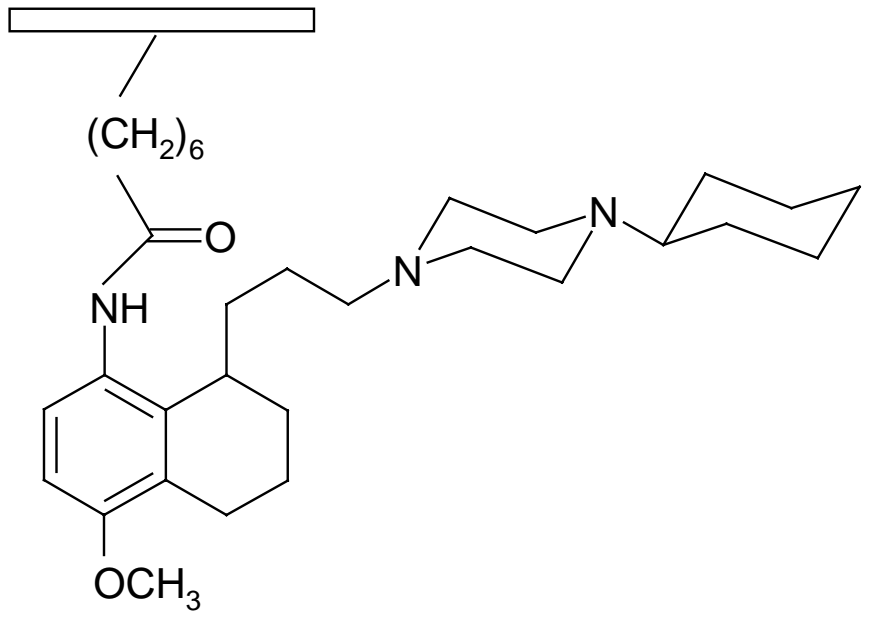

Isolation and characterization of histone proteins from human SK-N-SH neuroblastoma cell line by coupled stationary phase.

\section{Appendix}

\begin{tabular}{|c|c|c|c|c|c|c|}
\hline \multirow{3}{*}{ Compound } & \multicolumn{6}{|c|}{ Elemental analyses } \\
\cline { 2 - 7 } & \multicolumn{3}{|c|}{ Calculated } & \multicolumn{3}{c|}{ Found } \\
& $\mathbf{C \%}$ & $\mathbf{H \%}$ & $\mathbf{N \%}$ & $\mathbf{C \%}$ & $\mathbf{H \%}$ & $\mathbf{N \%}$ \\
\hline $4^{\boldsymbol{a}}$ & 60.47 & 8.77 & 8.81 & 60.54 & 8.30 & 8.42 \\
\hline
\end{tabular}

${ }^{a} \mathrm{C}_{24} \mathrm{H}_{39} \mathrm{~N}_{3} \mathrm{O}$ x $2.5 \mathrm{HCl} ; \mathrm{Mp}: 269-274{ }^{\circ} \mathrm{C}$. 


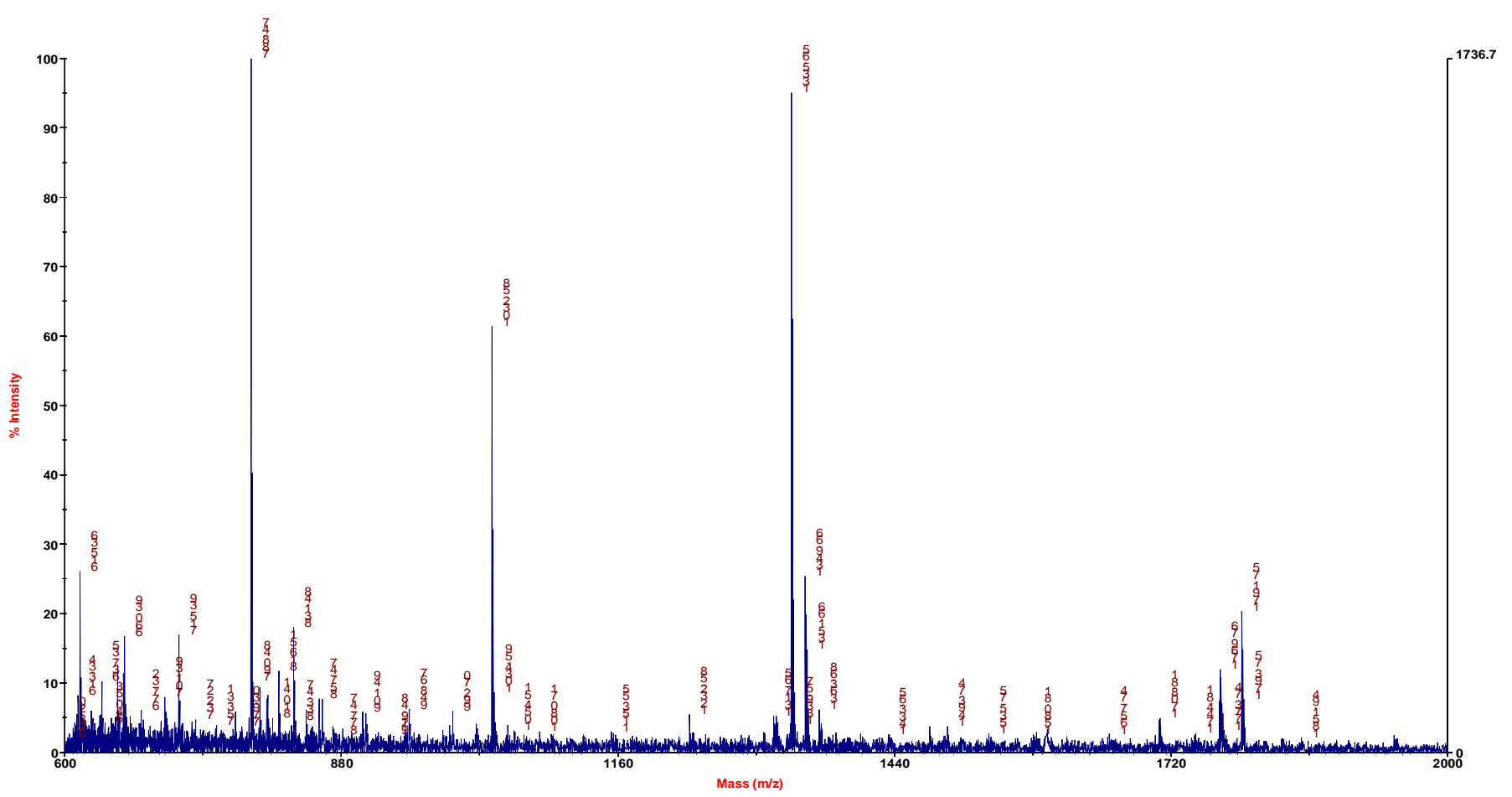




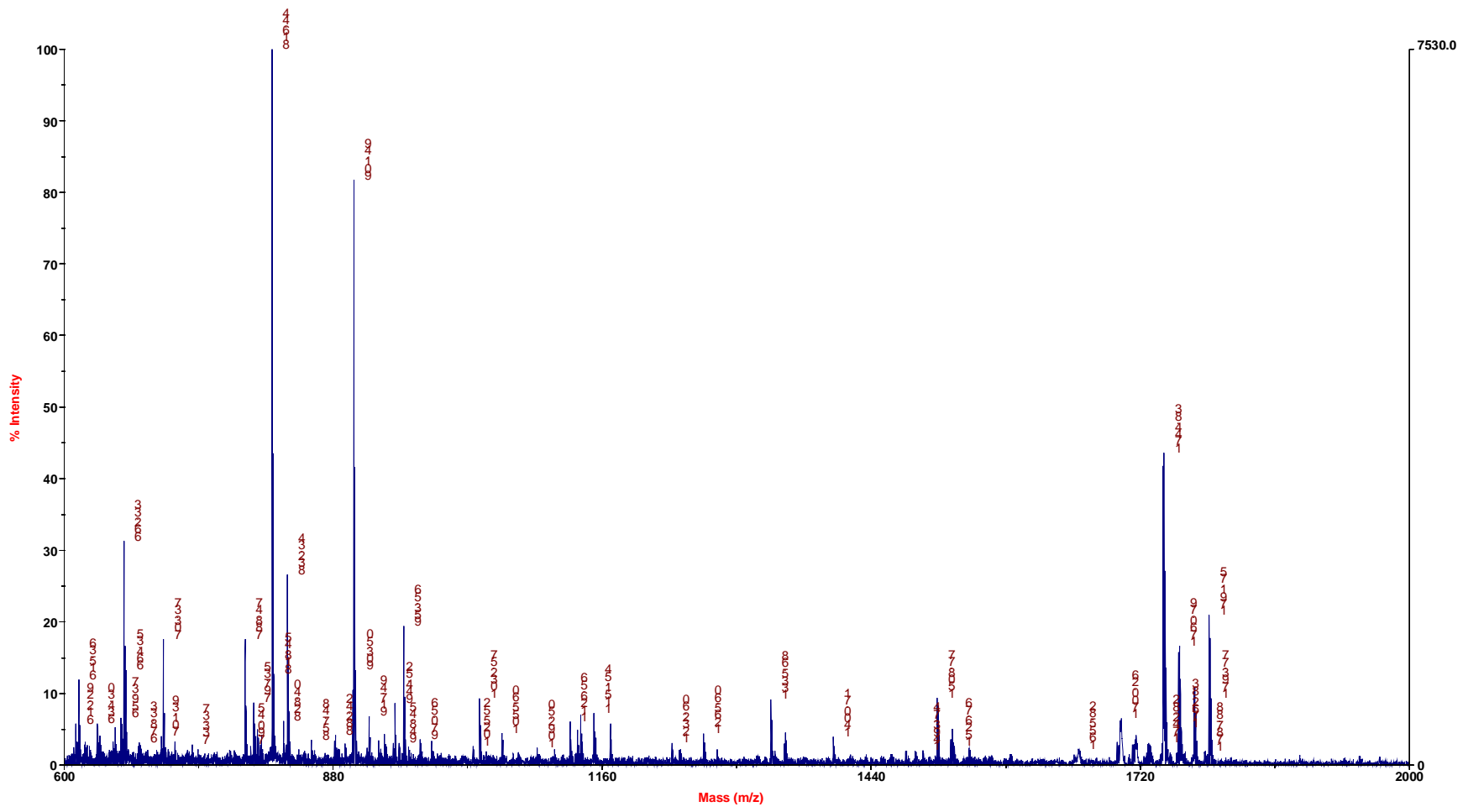




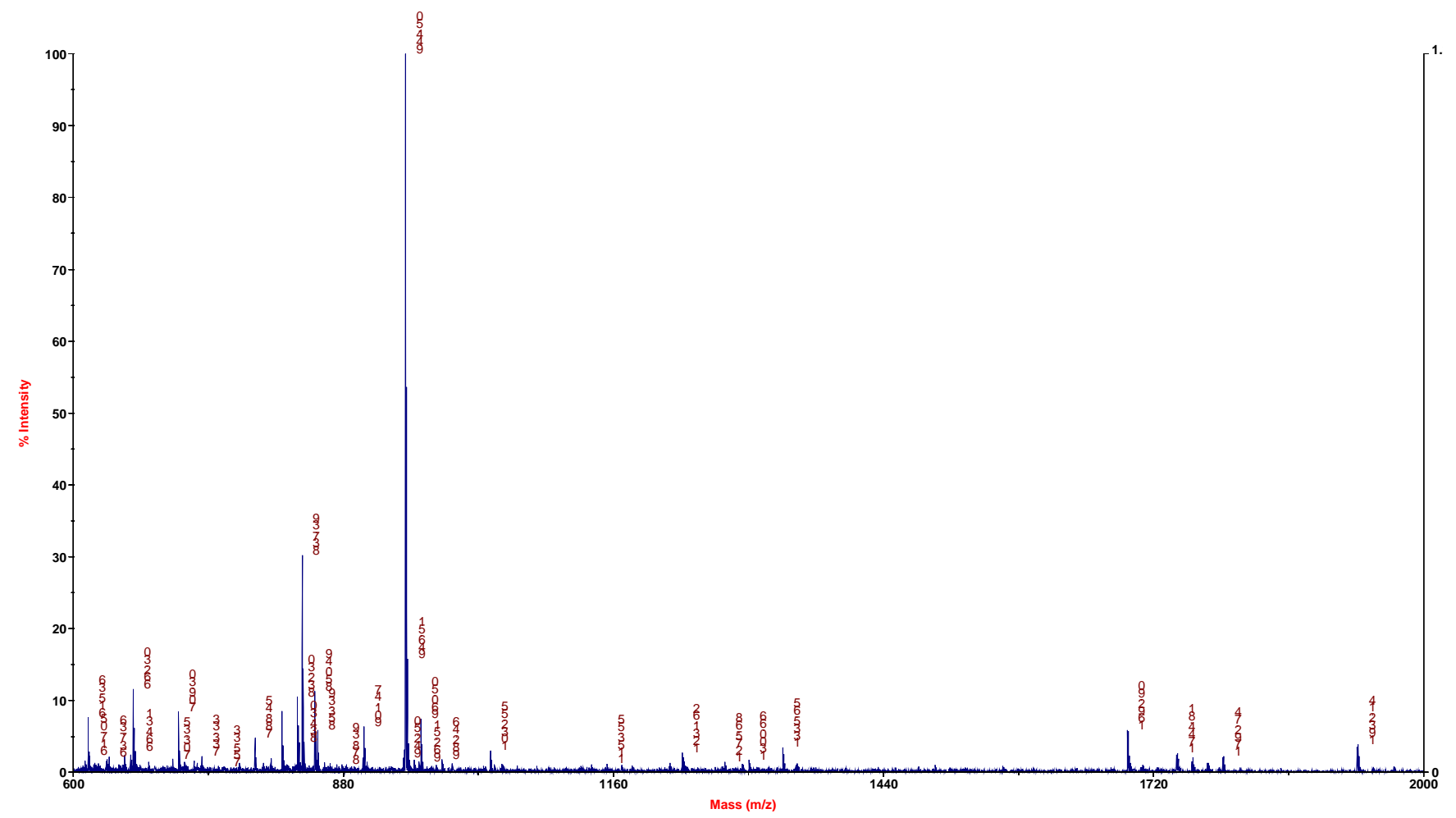




\section{Search Parameters and protein identification results}

\section{Protein band 15A}

gi|51859376 H3 histone, family 3A [Homo sapiens] Mass: 15333 Score: 113

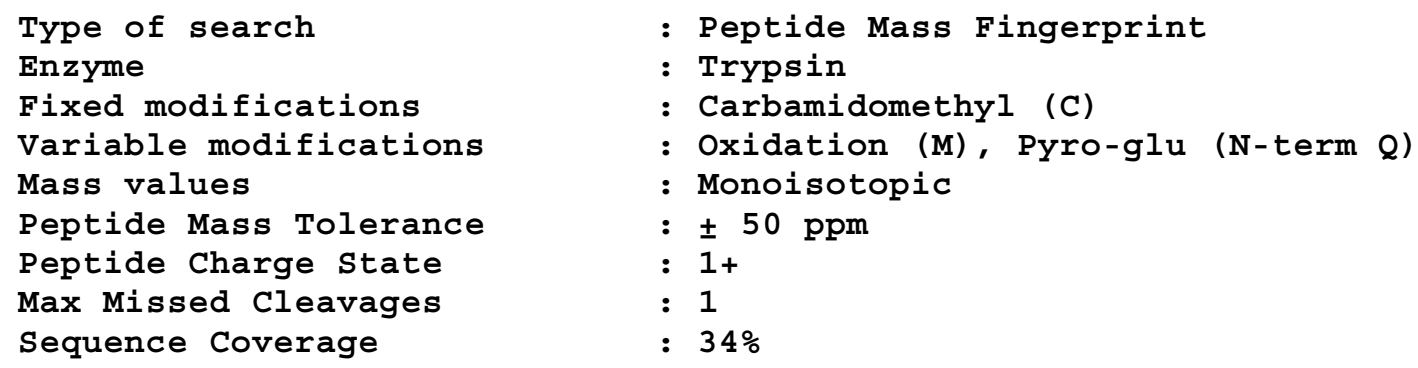

Matched peptides shown in Bold Red

1 MARTKQTALK STGGKAPRKQ LATKAARKSA PSTGGVKKPH RYRPGTVALR

51 EIRRYQKSTE LLIRKLPFQR LVREIAQDFK TDLRFQSAAI GALQEASEAY 101 LVGLFEDTNL CAIHAKRVTI MPKDIQLARR IRGERA

$\begin{array}{rrrrrrl}\text { Start - End } & \text { Observed } & \text { Mr(expt) } & \text { Mr(calc) } & \text { Delta } & \text { Miss } & \text { Sequence } \\ 42-50 & 1032.58 & 1031.57 & 1031.59 & -0.01 & 0 & \text { YRPGTVALR } \\ 58-64 & 831.48 & 830.47 & 830.49 & -0.01 & 0 & \text { STELLIR } \\ 65-70 & 788.47 & 787.46 & 787.47 & -0.01 & 1 & \text { KLPFQR } \\ 66-70 & 660.39 & 659.38 & 659.38 & 0.01 & 0 & \text { LPFQR } \\ 74-84 & 1335.65 & 1334.64 & 1334.68 & -0.04 & 1 & \text { EIAQDFKTDLR } \\ 117-123 & 844.49 & 843.48 & 843.50 & -0.02 & 1 & \text { RVTIMPK } \\ 117-123 & 860.54 & 859.53 & 859.49 & 0.04 & 1 & \text { RVTIMPK Oxidation (M) } \\ 124-129 & 715.39 & 714.38 & 714.40 & -0.02 & 0 & \text { DIQLAR } \\ 124-130 & 871.49 & 870.48 & 870.50 & -0.02 & 1 & \text { DIQLARR }\end{array}$

\section{Protein band 15B}

gi 1568557 histone H2B [Homo sapiens] Mass: 13928 Score: 126

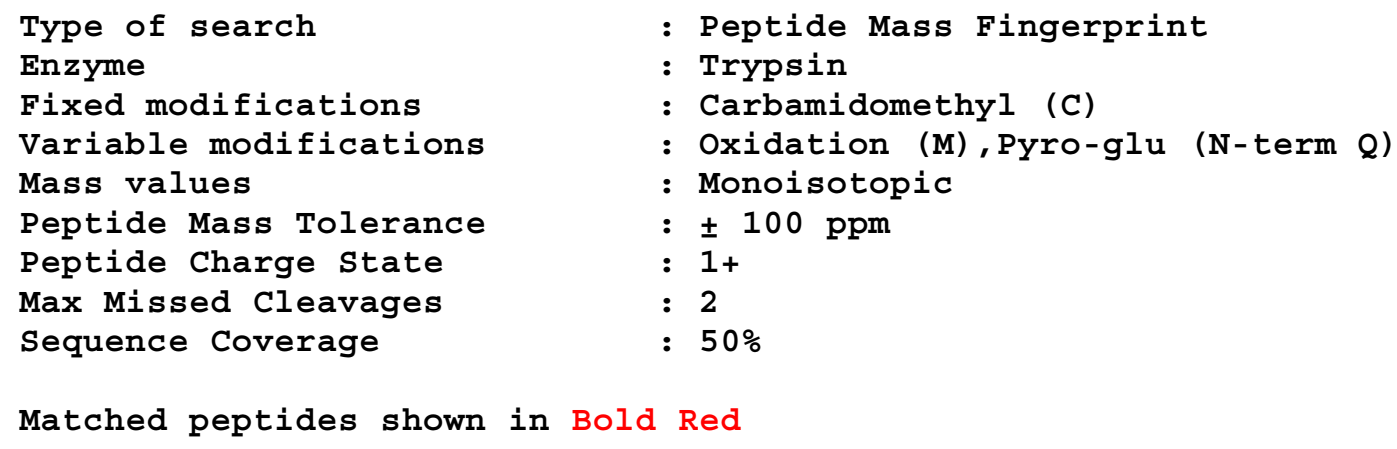

Matched peptides shown in Bold Red 
51 PDTGISSKAM GIMNSFVNDI FERIAGEASR LAHYNKRSTI TSREIQTAVR

101 LLLPGELAKH AVSEGTKAVT KYTSSK

\begin{tabular}{rlrllll} 
Start - End & Observed & \multicolumn{2}{c}{ Mr (expt) } & Mr (calc) & Delta & \multicolumn{2}{l}{ Miss Sequence } \\
$33-44$ & 1522.85 & 1521.84 & 1521.78 & 0.06 & 2 & TRKESYSVYVYK \\
$35-44$ & 1265.68 & 1264.67 & 1264.63 & 0.04 & 1 & KESYSVYVYK \\
$36-44$ & 1137.58 & 1136.57 & 1136.54 & 0.03 & 0 & ESYSVYVYK \\
$45-58$ & 1508.86 & 1507.85 & 1507.84 & 0.02 & 1 & VLKQVHPDTGISSK \\
$48-58$ & 1151.62 & 1150.61 & 1150.56 & 0.05 & 0 & QVHPDTGISSK PYrO-glu (Q) \\
$48-58$ & 1168.66 & 1167.65 & 1167.59 & 0.06 & 0 & QVHPDTGISSK \\
$59-73$ & 1743.93 & 1742.92 & 1742.81 & 0.11 & 0 & AMGIMNSFVNDIFER \\
$81-87$ & 901.56 & 900.55 & 900.49 & 0.06 & 1 & LAHYNKR \\
$94-100$ & 816.51 & 815.50 & 815.45 & 0.05 & 0 & EIQTAVR \\
$101-109$ & 953.64 & 952.63 & 952.60 & 0.04 & 0 & LLLPGELAK
\end{tabular}

Protein band $15 \mathrm{C}$

gi|70686 histone H2A.5 - [Homo sapiens] $\quad$ Mass: $14047 \quad$ Score: 110

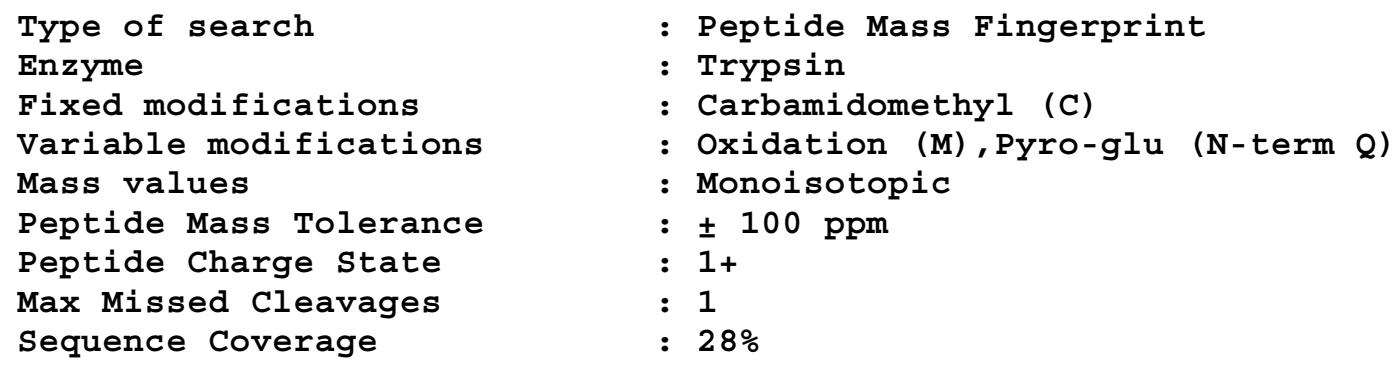

Matched peptides shown in Bold Red

1 SGRGKQGGKA RAKAKTRSSR AGLQFPVGRV HRLLRKXXYS ERVGAGAPVY

51 LAAVLEYLTA EILELAGNAA RDNKKTRIIP RHLQLAIRND EELNKLLGRV

101 TIAQGGVLPN IQAVLLPKKT ESHHKAKGK

\begin{tabular}{|c|c|c|c|c|c|c|c|}
\hline start & - End & Observed & $\operatorname{Mr}(\exp t)$ & $\operatorname{Mr}(c a l c)$ & Delta & Miss & Sequence \\
\hline 21 & -29 & 944.60 & 943.59 & 943.52 & 0.07 & 0 & AGLQFPVGR \\
\hline 21 & -32 & 1336.76 & 1335.75 & 1335.75 & 0.00 & 1 & AGLQF PVGRVHR \\
\hline 82 & -88 & 850.59 & 849.58 & 849.52 & 0.06 & 0 & HLQLAIR \\
\hline 82 & -95 & 1693.05 & 1692.04 & 1691.90 & 0.15 & 1 & HLQLAIRNDEELNK \\
\hline 89 & -99 & 1300.80 & 1299.79 & 1299.68 & 0.11 & 1 & NDEELNKLLGR \\
\hline 119 & -125 & 866.53 & 865.52 & 865.44 & 0.08 & 1 & KTESHHK \\
\hline
\end{tabular}


gi 356168 Histone H1 [Homo sapiens] Mass: 22566 Total score: 324

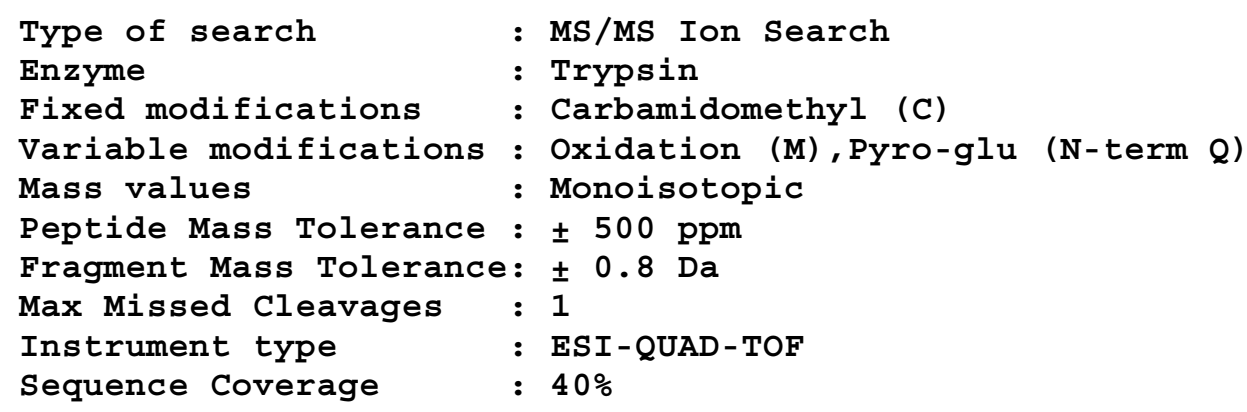

Matched peptides shown in Bold Red

1 MSETAPAETA TPAPVEKSPA KKKATKKAAG AGAAKRKATG PPVSELITKA VAASKERNGL

61 SLAALKKALA AGGYDVEKNN SRIKLGLKSL VSKGTLVQTK GTGASGSFKL NKKAASGEAK

121 PKAKKAGAAK AKKPAGATPK KAKKAAGAKK AVKKTPKKAK KPAAAGVKKV AKSPKKAKAA

181 AKPKKATKSP AKPKAVKPKA AKPKAAKPKA AKPKAAKAKK AAAKKK

\begin{tabular}{|c|c|c|c|c|c|c|c|c|}
\hline start & - End & Observed & $\operatorname{Mr}(\exp t)$ & $\operatorname{Mr}(\mathrm{calc})$ & Delta & Miss & SCORE & Sequence \\
\hline 58 & -67 & 443.83 & 885.65 & 885.53 & 0.13 & 0 & 59 & NGLSLAALK \\
\hline 58 & -68 & 507.87 & 1013.73 & 1013.62 & 0.10 & 1 & 18 & NGLSLAALKK \\
\hline 69 & -78 & 547.36 & 1092.70 & 1092.55 & 0.15 & 0 & 40 & ALAAGGYDVEK \\
\hline 189 & - 199 & 575.95 & 1149.89 & 1149.72 & 0.17 & 1 & 8 & SPAKPKAVKPK \\
\hline 102 & -112 & 583.90 & 1165.79 & 1165.61 & 0.18 & 1 & 8 & GTGASGSFKLNK \\
\hline 38 & -49 & 606.90 & 1211.78 & 1211.68 & 0.10 & 0 & 42 & ATGPPVSELITK \\
\hline 89 & -100 & 420.98 & 1259.91 & 1259.74 & 0.16 & 1 & 35 & SLVSKGTLVQTK \\
\hline 37 & -49 & 670.99 & 1339.96 & 1339.77 & 0.19 & 1 & (11) & KATGPPVSELITK \\
\hline 37 & -49 & 447.67 & 1339.98 & 1339.77 & 0.21 & 1 & 33 & KATGPPVSELITK \\
\hline 68 & -82 & 522.31 & 1563.90 & 1563.76 & 0.14 & 1 & 64 & ALAAGGYDVEKNNSR \\
\hline 68 & -82 & 782.99 & 1563.96 & 1563.76 & 0.20 & 1 & (59) & ALAAGGYDVEKNNSR \\
\hline 2 & -17 & 800.01 & 1598.02 & 1597.78 & 0.23 & 0 & 17 & SETAPAETATPAPVEK \\
\hline
\end{tabular}




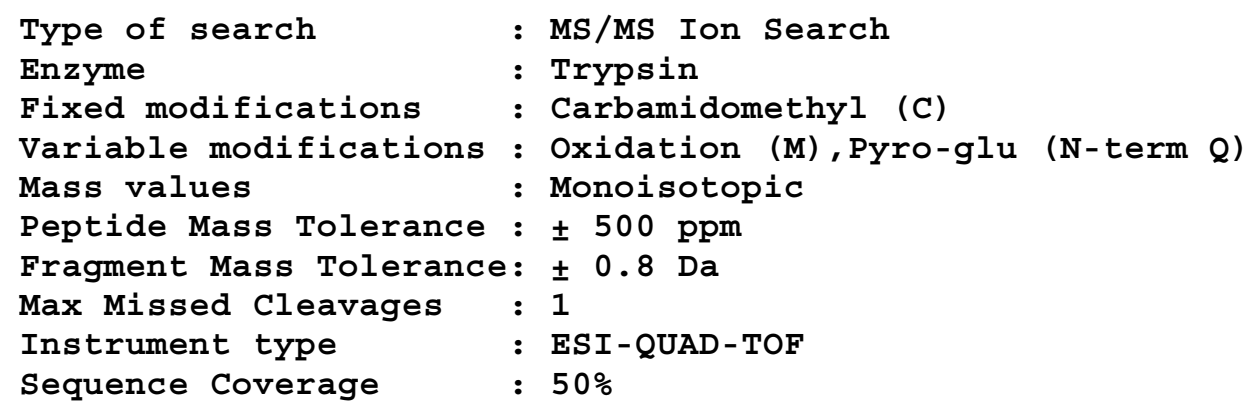

Matched peptides shown in Bold Red

1 MAVQISKKRK FVADGIFKAE LNEFLTRELA EDGYSGVEVR VTPTRTEIII

51 LATRTQNVLG EKGRRIRELT AVVQKRFGFP EGSVELYAEK VATRGLCAIA 101 QAESLRYKLL GGLAVRRACY GVLRFIMESG AKGCEVVVSG KLRGQRAKSM 151 KFVDGLMIHS GDPVNYYVDT AVRHVLLRQG VLGIKVKIML PWDPTGKIGP 201 KKPLPDHVSI VEPKDEILPT TPISEQKGGK PEPPAMPQPV PTA

$\begin{array}{lcc}\text { Delta } & \text { Miss } & \text { SCORE } \\ 0.04 & 0 & 15 \\ 0.03 & 0 & 47 \\ 0.06 & 0 & 25 \\ 0.02 & 1 & 7 \\ 0.04 & 0 & 52 \\ 0.07 & 0 & 17 \\ 0.09 & 0 & 10 \\ 0.03 & 0 & 42 \\ 0.13 & 1 & 15 \\ 0.03 & 0 & 52 \\ 0.00 & 0 & 11 \\ 0.08 & 0 & 10\end{array}$

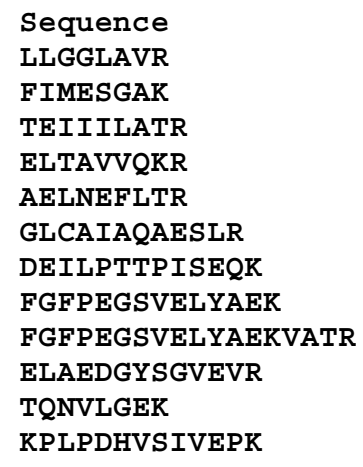

P16403

Type of search

Enzyme

Fixed modifications

Variable modifications

Mass values
: MS/MS Ion Search

: Trypsin

: Carbamidomethyl (C)

: Oxidation (M) , Pyro-glu (N-term Q)

: Monoisotopic 
Peptide Mass Tolerance : $\pm 500 \mathrm{ppm}$

Fragment Mass Tolerance: $\pm 0.8 \mathrm{Da}$

Max Missed Cleavages : 1

Instrument type : ESI-QUAD-TOF

Sequence Coverage : $23 \%$

Matched peptides shown in Bold Red

1 SETAPAAPAA APPAEKAPVK KKAAKKAGGT PRKASGPPVS ELITKAVAAS

51 KERSGVSLAA LKKALAAAGY DVEKNNSRIK LGLKSLVSKG TLVQTKGTGA

101 SGSFKLNKKA ASGEAKPKVK KAGGTKPKKP VGAAKKPKKA AGGATPKKSA

151 KKTPKKAKKP AAATVTKKVA KSPKKAKVAK PKKAAKSAAK AVKPKAAKPK

201 VVKPKKAAPK KK

$\begin{array}{cccccccl}\text { Start - End } & \text { Observed } & \text { Mr (expt) } & \text { Mr calc) } & \text { Delta } & \text { Miss } & \text { SCORE } & \text { Sequence } \\ 33-45 & 442.95 & 1325.84 & 1325.76 & 0.09 & 1 & 26 & \text { KASGPPVSELITK } \\ 64-74 & 554.31 & 1106.61 & 1106.56 & 0.05 & 0 & 48 & \text { ALAAAGYDVEK } \\ 64-78 & 789.92 & 1577.84 & 1577.78 & 0.06 & 1 & 14 & \text { ALAAAGYDVEKNNSR } \\ 54-62 & 423.27 & 844.52 & 844.50 & 0.02 & 0 & 34 & \text { SGVSLAALK } \\ 85-96 & 420.95 & 1259.81 & 1259.74 & 0.07 & 1 & 36 & \text { SLVSKGTLVQTK } \\ 34-45 & 599.86 & 1197.71 & 1197.66 & 0.05 & 0 & 3 & \text { ASGPPVSELITK }\end{array}$

